\title{
Effect of liquid viscosity on the aerodynamic breakup of non-spherical droplets
}

\author{
Konstantinos Bergeles ${ }^{1, *}$, Georgios Charalampous ${ }^{1}$, Yannis Hardalupas ${ }^{1}$ and Alex M. Taylor ${ }^{1}$ \\ ${ }^{1}$ Mechanical Engineering Department, Imperial College London, UK \\ *Corresponding author: k.bergeles12@imperial.ac.uk
}

\begin{abstract}
This paper studies the effect of liquid viscosity on the atomisation regimes of initially spherical and non-spherical droplets and also kinematic characteristics of non-spherical droplets. The droplets consisted of water-glycerol solutions with viscosities ranging from 6.3 to $697 \mathrm{mPas}$, and the initial aspect ratio was $1<\mathrm{AR}<2$. The range of $W e$ number was from 10 to 200 and of the $O h$ number from 0.01 to approximately 4 . The experimental findings showed that the equivalent $W e_{\mathrm{eq}}$ and $O h_{\mathrm{eq}}$ numbers, proposed in previous work [1] for water droplets, are also applicable to spherical and non-spherical droplets in the range of $O h$ numbers of this study in order to classify the breakup regimes on the existing morphological charts. The kinematic characteristics of the centre of mass for droplets with $A R=1.2$ are evaluated and the role of viscosity examined in the no breakup and bag-stamen regimes.
\end{abstract}

\section{Keywords}

non-spherical, droplet, breakup, aerodynamic

\section{Introduction}

Understanding the physics and nature of liquid droplet-air interaction, investigating the droplet internal and external flow and capturing droplet deformation and breakup is of importance in various scientific fields and technological applications [2]. In some technological applications, such as automotive sprays, the need is to avoid the generation of the largest droplets. Although their number density is low, these sizes carry a disproportionate fraction of the injected fuel, which evaporates slowly, and follow 'ballistic' trajectories resulting in locally overly rich mixtures, associated with undesirable emission characteristics.

The literature on experimental droplet atomisation assumes an initial spherical droplet. In other words, most research classifies and investigates the breakup modes under morphological regimes treating the droplet as a sphere. However, in regions of intense atomisation, as found in the interior of the crankcase, the technological motivator of the present work, droplets are known to have an initial non-spherical shape before the interaction with the flow inside the crankcase.

According to Hinze [3] droplets droplet deformation occurs as the value of $W e$ becomes greater than one $(W e>1)$. The external aerodynamic force acting on the droplet's surface, which scales as $\rho u^{2}$ becomes larger than the counteracting force owing to the surface tension $\sigma / d_{0}$ of the liquid. The deformation evolves to breakup at the critical value of $W e_{\text {crit }}=12$ number. In cases where $W e \approx W e_{\text {crit }}$ number simple breakup mechanism occurs, while for increasing $W e$ number, the disintegration of the droplet becomes a more chaotic atomisation process.

Pilch and Erdman [4] performed experiments to quantify characteristic variables of the breakup process, classifying the breakup modes. For increasing $W e$ number the atomisation regimes found were the vibrational, bag, bagstamen, sheet stripping and catastrophic breakup as shown in Figure 1. The normalised displacement $x_{d} *$ (Eq. (1)), velocity $u_{d}^{*}$ (Eq. (2)) and acceleration $a_{d}^{*}$ (Eq. (3)) of the centre of mass of the droplet were also presented. The study modelled the droplet as a rigid sphere, neglecting mass loss during the atomisation, as also the droplet velocity relative to the flow assuming an average, constant drag coefficient $C_{d}$ throughout the breakup process.

$$
\begin{aligned}
& x_{d}^{*}=\frac{x_{d}}{d_{0}}=\frac{3}{8} C_{d}\left(t^{*}\right)^{2} \\
& u_{d}^{*}=\frac{u_{d}}{u_{g} \cdot \epsilon^{0.5}}=\frac{3}{4} C_{d} t^{*} \\
& \alpha_{d}^{*}=\frac{\alpha_{d} \cdot d_{0}}{u_{g}^{2} \cdot \epsilon}=\frac{3}{4} C_{d}
\end{aligned}
$$

, where $x_{d}, u_{d}, a_{d}$ are the dimensioned displacement, velocity and acceleration of the centre of mass of the droplet. The timescale $t^{*}$ of the atomisation phenomenon (Eq. (4)) equals to:

$$
t^{*}=\frac{t \cdot u_{g} \epsilon^{1 / 2}}{d_{0}},
$$

where $\epsilon$ is the density ratio $\rho_{g} / \rho_{l}, t$ is the dimensioned time and $C_{d}$ is the droplet drag coefficient which remains constant during the atomisation and equal to $C_{d}=2.5$ and $C_{d}=1.7$ for compressible and incompressible flows respectively. 


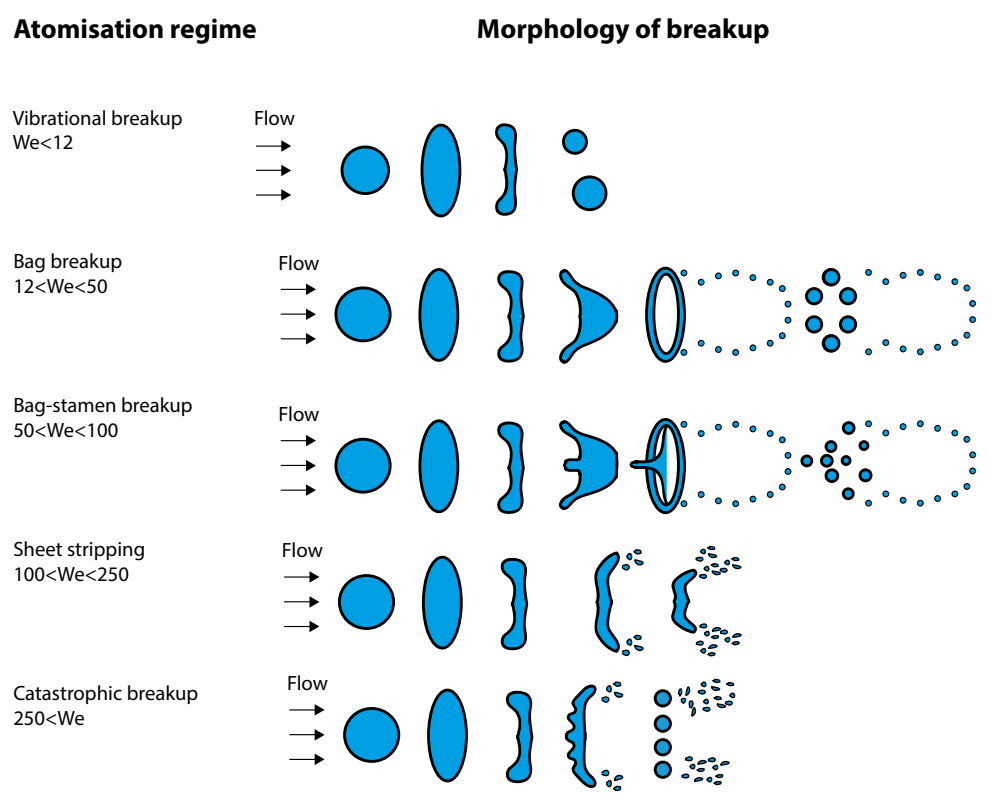

Figure 1. Morphological classification of breakup modes based on $W e$ number as derived by Pilch and Erdman [4]

In addition Pilch and Erdman [4] proposed a third degree polynomial to express the droplet displacement $x_{d} *$ (Eq. (5)), velocity $u_{d}^{*}$ (Eq. (6)) and acceleration $a_{d}^{*}$ (Eq. (7)) using the empirical constant $B$ to provide a correct initial behaviour.

$$
\begin{aligned}
& x_{d}^{*}=\frac{x_{d}}{d_{0}}=\frac{3}{8} C_{d}\left(t^{*}\right)^{2}+B\left(t^{*}\right)^{3} \\
& u_{d}^{*}=\frac{u_{d}}{u_{g} \cdot \epsilon^{0.5}}=\frac{3}{4} C_{d} t^{*}+3 B\left(t^{*}\right)^{2} \\
& \alpha_{d}^{*}=\frac{\alpha_{d} \cdot d_{0}}{u_{g}^{2} \cdot \epsilon}=\frac{3}{4} C_{d}+6 B t^{*}
\end{aligned}
$$

with $C_{\mathrm{d}}=1, B=0.116$ for compressible flow and $C_{d}=0.5, B=0.0758$ for incompressible flow. The correlation assumes a constant drag coefficient $C_{\mathrm{d}}$ of a rigid constant-mass sphere. the change of the frontal area of the droplet due to deformation is not taken into account. Moreover, the equations are valid for the period of time until the velocity of the droplet is $60 \%$ of the velocity of the flow.

The transition between two breakup regimes has been found to be a function of the $W e$ and $O h$ number and independent of the density ratio or the Re number. These transitional $W$ e numbers have been quantified by Brodkey [5] in Eq. (8) and Gelfand [6] in Eq. (9).

$$
\begin{aligned}
& W e_{\text {crit }}=W e_{\text {crit }, 0}\left(1+1.077 O h^{1.6}\right) \\
& W e_{\text {crit }}=W e_{\text {crit }, 0}\left(1+1.5 O h^{0.74}\right)
\end{aligned}
$$

, where $W e_{\text {crit }}$ is the transitional $W e$ number for increased $O h$ number and $W e_{\text {crit }, 0}$ is the transitional $W e$ number for $O h \rightarrow 0$ respectively.

However a gap exists in the literature of the droplet atomisation as all the previous analytical work and interpretation of experimental data assumed initial spherical droplets.

\section{Experimental arrangement and measurement technique}

The experimental arrangement is shown in Figure 2. The main components are a Tec5 ultrasonic levitator which is used to levitate individual droplets and control their sphericity, a Photron CMOS high-speed camera for the imaging of the breakup and a nozzle controlled by a high-speed solenoid valve which produces the cross-flow that atomises the droplet.

Droplet levitation: An ultrasonic wave is emitted from the transducer of the levitator, which is reflected back to its source by a reflector, creating a vertical standing wave. A single droplet with diameter of the order of one millimetre is 


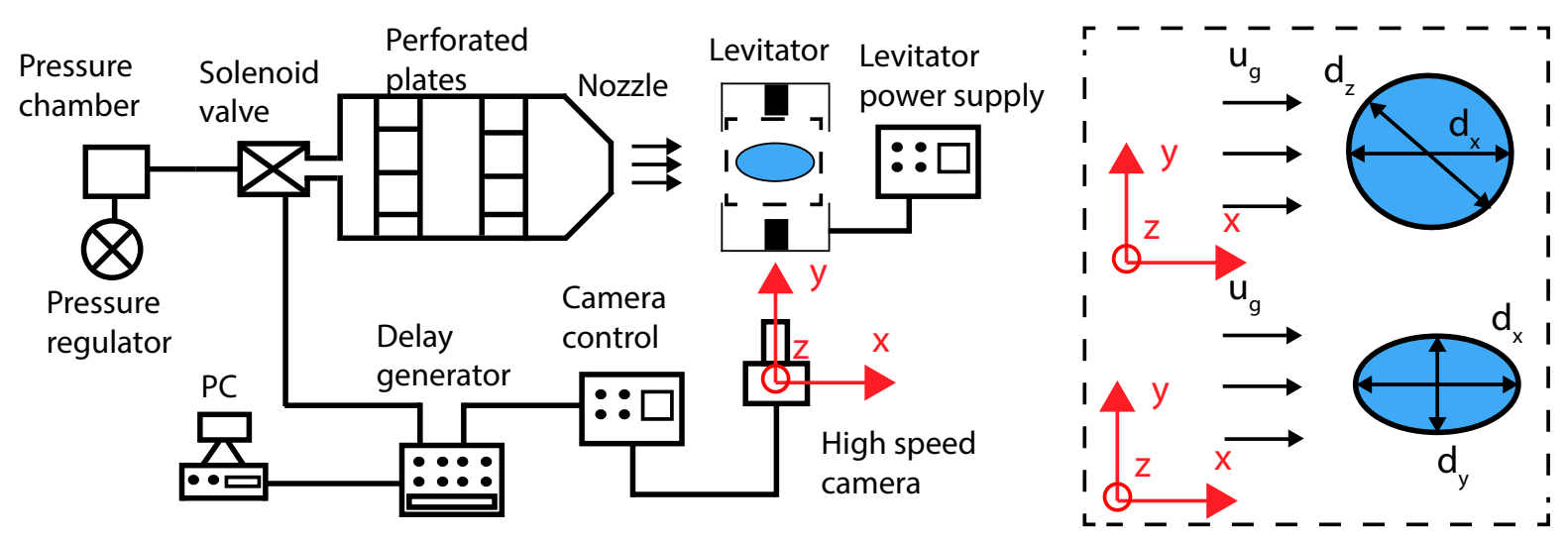

Figure 2. Experimental arrangement of the aerodynamic breakup study of spherical and non-spherical droplets

Table 1. Physical properties of glycerol-water solutions at $20^{\circ} \mathrm{C}$ ambient temperature

\begin{tabular}{lcccc}
\hline $\begin{array}{l}\text { Name } \\
\text { (\% glycerol) }\end{array}$ & $\begin{array}{c}\text { Dynamic viscosity } \\
\mu_{l}[\mathrm{mPa}]\end{array}$ & $\begin{array}{c}\text { Kinematic viscosity } \\
\nu_{l}\left[\mathrm{~mm}^{2} / \mathrm{s}\right]\end{array}$ & $\begin{array}{c}\text { Density } \\
\rho_{l}\left[\mathrm{~kg} / \mathrm{m}^{3}\right]\end{array}$ & $\begin{array}{c}\text { Surface tension } \\
\sigma[\mathrm{mN} / \mathrm{m}]\end{array}$ \\
\hline \hline & & & & \\
Mix-1 (50\%) & 6.386 & 5.674 & 1125.5 & 68.5 \\
Mix-2 (80\%) & 58.96 & 48.839 & 1207.3 & 65.5 \\
Mix-3 (97\%) & 697 & 557.15 & 1251.0 & 63.0 \\
\hline
\end{tabular}

placed at a pressure node and levitated until aerodynamically atomised from a high speed air stream. The sphericity of the droplet is controlled by adjusting the amplitude of the ultrasonic waves, permitting the transformation of a spherical droplet into an oblate spheroidal droplet.

The acoustic pressure induced on the upper and lower pole of the droplet plays a minor role during the deformation and atomisation process. For a millimetre droplet, the lift force generated by the acoustic pressure balances the gravitational force and caused a total pressure within the droplet to increase by approximately $10 \mathrm{~Pa}$. The acoustic pressure is the cause of transformation of the spherical droplet shape into an oblate spheroidal shape, due to surface tension, approximately $70 \mathrm{~Pa}$ for the maximum $A R$. The minimum dynamic pressure of the flow is $500 \mathrm{~Pa}$, therefore the contribution of the maximum acoustic pressure to the droplet atomisation is an order of magnitude lower compared to the minimum dynamic pressure of the flow. As a result, the role of the acoustic pressure on the atomisation is expected to be negligible.

Gas flow: The droplets were impulsively accelerated by a sudden gust of air, which was released from a pressure chamber by a fast solenoid valve. The cross-flow consisted of filtered air at room temperature $20^{\circ} \mathrm{C}$. The air jet flow was ejected through a nozzle with $10 \mathrm{~mm}$ diameter. The velocity at the exit of the nozzle was measured using a Dantec hotwire device to examine the uniformity and repeatability of the velocity profile. The velocity profile of the flow as a function of time is close to a step function with accelerations ranging from 1928 up to $13 \cdot 10^{3} \mathrm{~m} / \mathrm{s}^{2}$ over the range of experiments. The coefficient of variance of the acceleration is lower than $8 \%$ for all operating conditions.

The maximum and mean velocity value of the flow were measured at four distinct points at a radius of $2 \mathrm{~mm}$ and at the centre of the nozzle. The measurements, show an almost uniform velocity profile with a standard deviation well below 3\%, thus the droplet atomisation occurs under a uniform velocity profile. However, for low air velocities, discrepancies in the flow are larger, but still within an acceptable range with maximum standard deviation of around $4.3 \%$.

High-speed imaging: The shadowgraphic technique is employed for the visualisation of the droplet breakup. A Photron high-speed CMOS camera records the droplet breakup at a frame rate of $20 \mathrm{kHz}$. The spatial resolution of the images is $512 \times 256$ pixels with $5 \mu$ s exposure time. A commercial Nikon lens ( $f$-number $f / \#=2.8)$ with appropriate extension tubes and a teleconverter (TELEPLUS MC7 AF 2.0X DGX) are used leading to an image magnification of $12.3 \mu \mathrm{m} /$ pixel. The imaged area of the camera is $6.2 \mathrm{~mm} \times 3.1 \mathrm{~mm}$ in the xy plane as shown in Figure 2 .

Due to the high acquisition rate, the droplet breakup was temporally resolved. The camera was placed perpendicular to the flow direction and it was focused on the symmetry plane of the droplet. The synchronisation of the solenoid valve with the high-speed camera was achieved using a SRS 535 digital delay generator. Finally, a back illumination lamp of $1000 \mathrm{~W}$ was used and uniform illumination was accomplished with ground glass diffusers. The processing of the images was performed in Matlab environment using an in-house code and in ImageJ [7], an image manipulation software.

For the present study three glycerol-water solutions were produced and the viscosity measured using a viscometer. The physical properties were derived for $20^{\circ} \mathrm{C}$ ambient temperature and the characterisation error is within $2 \%$. Table 1 summarises the physical properties of the glycerol-water solutions. 


\section{Morphological classification of droplet breakup}

The breakup modes appearing for increasing $W e$ numbers are the no, bag, bag-stamen and sheet stripping breakup modes. These regimes change for increasing $O h$ numbers and it must be pointed out that the transition between breakup regimes is not a step function but it occurs gradually. Therefore the characterisation of a breakup could be difficult and rather arbitrary, especially between bag-stamen and sheet stripping breakup regimes and also for viscous liquids.

The most commonly used theoretical curves describing this transition of the breakup modes are produced by Brodkey [5] (Eq. (8)) and Gelfand [6] (Eq. (9)). According to these relations, a steep change in the transitional $W e$ numbers appears for higher $O h$ number $(O h>0.1)$. From this $O h$ number and onward the liquid viscous forces become dominant and an exponential change occurs to the transitional $W e$ numbers.

The morphological classification in the $O h-W e$ number plane of the present experimental data is shown in Figure 3. The derivation of the dimensionless numbers was done with the diameter $d_{0}$ based on droplet's volume. Overlapping regions appeared, especially in the bag and bag-stamen breakup regimes for Mix-1 (50\% glycerol) and Mix-2 (80\% glycerol) solutions.

The theoretical curve of Brodkey [5] and Gelfand [6] are also presented in Figure 3. The regime boundaries are approximately flat for $O h<0.1$; Gelfand's relation [6] produces higher transitional $W e$ numbers for lower $O h$ numbers than Brodkey's [5], however the latter one predicts a steeper change in the transitional $W e$ numbers. The theoretical curves of Brodkey [5] and Gelfand [6] predicting the breakup regime boundaries should be taken as a general rule.

For $O h>0.05$ the discrepancies of the two theoretical curves become significant, showing that the transition criteria of breakup regimes for increasing $\mathrm{Oh}$ are not well defined even for initially spherical droplets. The experimental data does not agree with the predicted transitional $W e$ numbers because the initial sphericity of the droplet has not taken into consideration by the theoretical curves.

Figure 4 is the representation of the breakup modes in $W e_{\mathrm{eq}}-O h_{\mathrm{eq}}$ plane. The derivation of the dimensionless numbers was done with the equivalent droplet diameter $d_{\text {eq }}$ (Eq. (11). The equivalent $W e_{e q}$ [1] (Eq. (10)) number for deformed droplet is:

$$
W e_{e q}=\frac{\rho_{g} u_{g}^{2} d_{\mathrm{eq}}}{\sigma}
$$

, where $d_{\text {eq }}$ is the equivalent diameter and defined in Eq. (11)

$$
d_{e q}=\frac{d_{x}^{2}}{d_{y}}
$$

, where $d_{x}, d_{y}$ are the stream-wise and cross-stream diameter of the droplet respectively. In the case of the spherical droplet, the equivalent diameter is equal to the diameter of the droplet $d_{0}$ based on its volume. In the same way the equivalent $O h_{e q}[1]$ is formulated (Eq. (12))

$$
O h_{e q}=\frac{\mu_{l}}{\sqrt{\rho_{l} \sigma d_{\mathrm{eq}}}}
$$

Figure 3 and Figure 4 map the breakup mode of both initially spherical and non-spherical droplets; however the initial sphericity of the droplets is not illustrated in the figures for clarity purposes. The introduction of $d_{\text {eq }}$ to the $O h$ number gives $O h_{\text {eq }}<O h$; in other words the equivalent diameter $d_{\text {eq }}$ "reduces" the effect of liquid viscosity (and as a consequence of the overall $O h$ number) to the transition of breakup modes. For the same $W e$ number, higher initial $d_{\text {eq }}$ leads to lower $O h_{\text {eq }}$ compared to a spherical droplet $\left(d_{0}, O h\right)$ resulting to a "higher" breakup regime. The combined effect of the equivalent $O h_{\mathrm{eq}}$ and $W e_{\mathrm{eq}}$ is to translate the experimental data upwards and to the left; meaning that if one is held constant the introduction of $d_{e q}$ leads to "higher" breakup regimes.

For the two least viscous solutions (50\% and $80 \%$ glycerol) the overlapping of the experiential data appears decreased compared to the $\mathrm{We}-\mathrm{Oh}$ plane representation. Additionally, both sets of experimental data are in better agreement with the two theoretical curves (Figure 8 and Figure 9).

The equivalent dimensionless groups $\left(W e_{\text {eq }}\right.$ and $\left.O h_{\text {eq }}\right)$ incorporate the initial non-sphericity of the droplets which lead to a better mapping of the experimental data than the conventional $W e$ and $O h$ numbers. For droplets with $O h>1$ ( $97 \%$ glycerol) the regions where breakup modes overlap remains. As the viscosity effects become dominant, the role of $O h$ number becomes important.

\section{Effect of liquid viscosity to the kinematic characteristics of droplets}

The displacement $x_{d}^{*}$, velocity $u_{d}^{*}$ and acceleration $\alpha_{d}^{*}$ of the centre of droplet's viewable area are presented as a function of dimensionless time $t^{*}$ for water and three glycerol-water solutions $(50 \%, 80 \%$ and $97 \%$ glycerol). The results of the study are split in two sections, for low and high $W e$ numbers, or in other words for no and bag-stamen breakup regimes. The aspect ratio $A R$ chosen for this study is $A R=1.2$. It could be achieved with all liquid with satisfactory repeatability leading to minimisation of the errors regarding the droplet's volume and initial $A R$; both of the errors are below $5 \%$. For the normalisation of the results the diameter $d_{0}$ is used. This is done in order to maintain the physical meaning of the kinematic characteristics. For example, an initially spherical droplet translates faster than an initially non-spherical. When $d_{\mathrm{eq}}$ is employed for the normalisation of the velocity this would not be the case for the non-dimensional velocity $u_{d}^{*}$. However, in the present paper, since the is constant $(A R=1.2)$, diameter $d_{\text {eq }}$ could be used as well as it is the same for all breakup cases presented. 


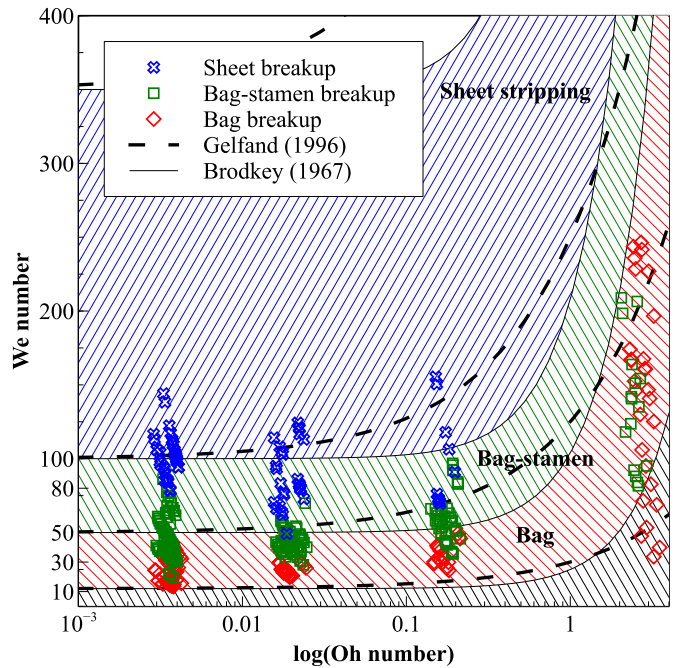

Figure 3. Morphological classification of breakup modes of spherical and non-spherical droplets consist of water and Mix-1, 2 and 3 (50\%, 80\% and 97\% glycerol) in $W e$-Oh plane derived with $d_{0}$

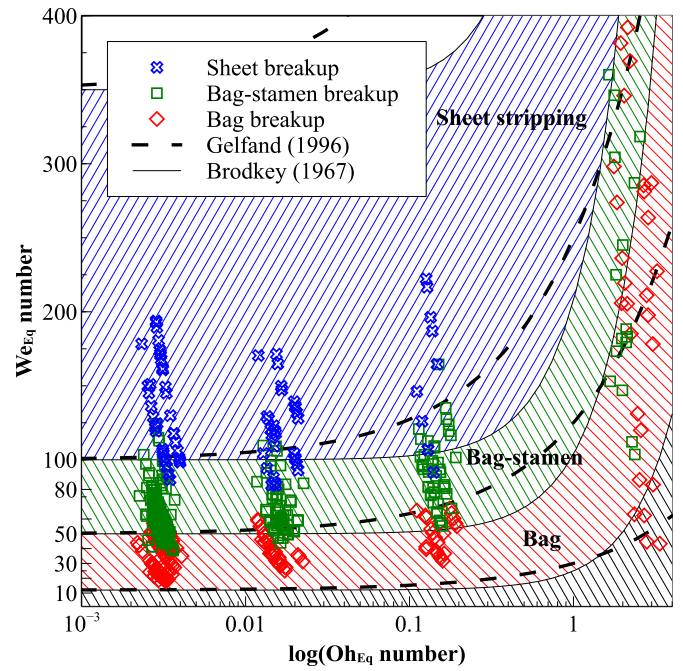

Figure 4. Morphological classification of breakup modes of spherical and non-spherical droplets consist of water and Mix-1, 2 and 3 (50\%, 80\% and $97 \%$ glycerol) in $W e_{\mathrm{eq}}-\mathrm{Oh}$ eq plane derived with $d_{\mathrm{eq}}$

Low $W e$ number - No breakup regime

The $W e$ number ranges from 10 to 13 with the $O h$ number being $3.410^{-3}, 0.02,0.19$ and 2.2 for increasing liquid viscosities. The diameter $d_{0}$ based on the droplet volume is $d_{0}=1.25 \pm 0.05 \mathrm{~mm}$ and the $A R=1.20 \pm 0.02 \mathrm{The}$ variation in the values for $W e$ number occurs due to the difference in the physical properties of the liquids, namely the density and the surface tension.

Figure $5 a$ presents a comparison of the non-dimensional displacement $x_{d}^{*}$ of the centre of viewable area of the droplet with dimensionless time $t^{*}$ for the four liquids (water and $50 \%, 80 \%$ and $97 \%$ glycerol) with the $3^{\text {rd }}$ degree fitting curve of Pilch and Erdman [4] which is independent of viscosity. The fitting curve generally under-predicts the displacement of the droplet and this is mainly due to the increased frontal area of the droplets compared to the spherical droplet assumed by the $3^{\text {rd }}$ degree fitting curve.

Figure $5 \mathrm{~b}$ shows the non-dimensional velocity $u_{d}^{*}$ of the centre of viewable area of the droplet. For glycerol-water solutions, after they achieve their maximum deformation $d_{\max }^{*}$, which occurs at $t_{\max }^{*}=1.18,2.55$ and 2.6 for $50 \%, 80 \%$ and $97 \%$ glycerol solutions respectively, they continue to translate with constant frontal area approximately equal to their maximum deformation.

Regarding the water droplet, its velocity $u_{d}^{*}$ oscillates due to the changes of the frontal area of the droplet. At $t_{\max }^{*}=1.07$ the water droplet achieves its maximum deformation $d_{\max }^{*}=1.42$, however at $t^{*}=2.1$ it becomes nearly spherical again. The reason why the oscillatory behaviour did not appear to that extent for the glycerol-water solutions is because the increased viscosity dampens the droplet oscillations. This oscillatory behaviour is not predicted by the $3^{\text {rd }}$ degree fitting curve. The velocities of the droplet as they exit the imaged area are $u_{d}^{*}=2.6,2.8$, 3.3 and 3.0 for increasing liquid viscosity and are directly related to the droplet frontal area.

The extent of the droplet's oscillatory behaviour decreases with the increase of the liquid viscosity as also does its effect on the translation behaviour of the droplet. Figure $5 c$ shows the temporal evolution of the normalised acceleration of the droplet $\alpha_{d}^{*}$. The data for the acceleration were derived by fitting a $5^{\text {th }}$ degree polynomial to the displacement data of the four liquids and taking the second derivative of the polynomial. A $5^{\text {th }}$ degree polynomial was used so that its second derivation was a third degree, which is able to predict the oscillation; the correlation coefficient is more than 0.95 regarding the displacement data. The bars in Figure $5 \mathrm{c}$ indicate the difference of the fitting curve value from the corresponding value of the experimental data for the acceleration $\alpha_{d}$.

The acceleration provides an overview of the behaviour of the droplet's frontal area with time; in particular the rate of change of the acceleration is related with the rate of change of the frontal area. As expected the water droplet accelerates faster than the rest of the droplets as it deforms faster due to its low viscosity. Additionally, the water and the least viscous glycerol-water solution ( $50 \%$ glycerol) are oscillating; Mix-2 ( $80 \%$ glycerol) oscillates but with a very small amplitude and finally no oscillation occurs for the most viscous case $(97 \%$ glycerol). The two least viscous droplets accelerate faster than the other two more viscous droplets. After that time the frontal area of the two droplet reduces and therefore the acceleration decreases; at time $t^{*}=1.6$ the two more viscous droplets, which did not oscillate, have higher acceleration than the least viscous droplets despite the fact that the latter ones have higher rate of increase of the frontal area. 


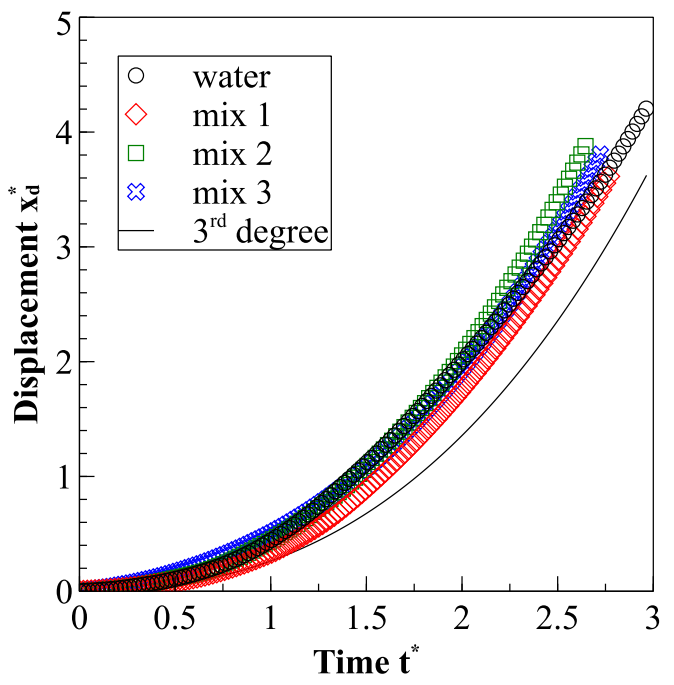

(a) Displacement $x_{d}^{*}$

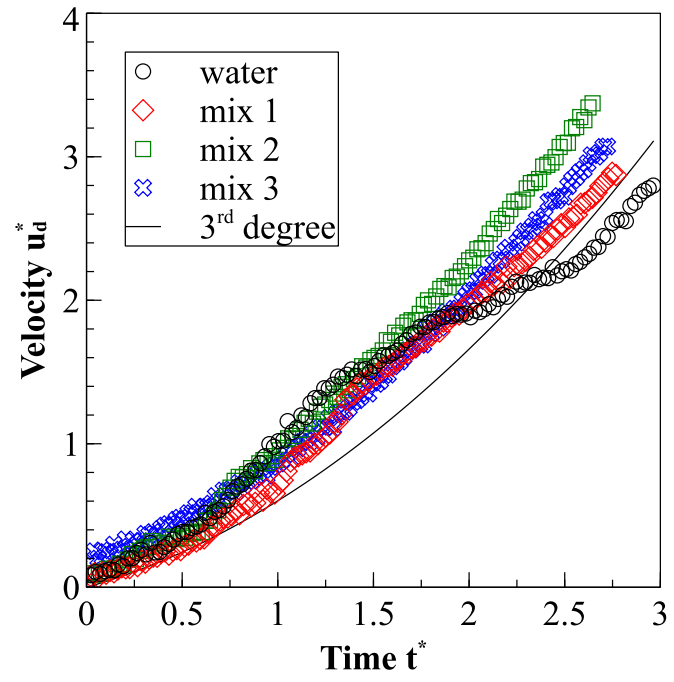

(b) Velocity $u_{d}^{*}$

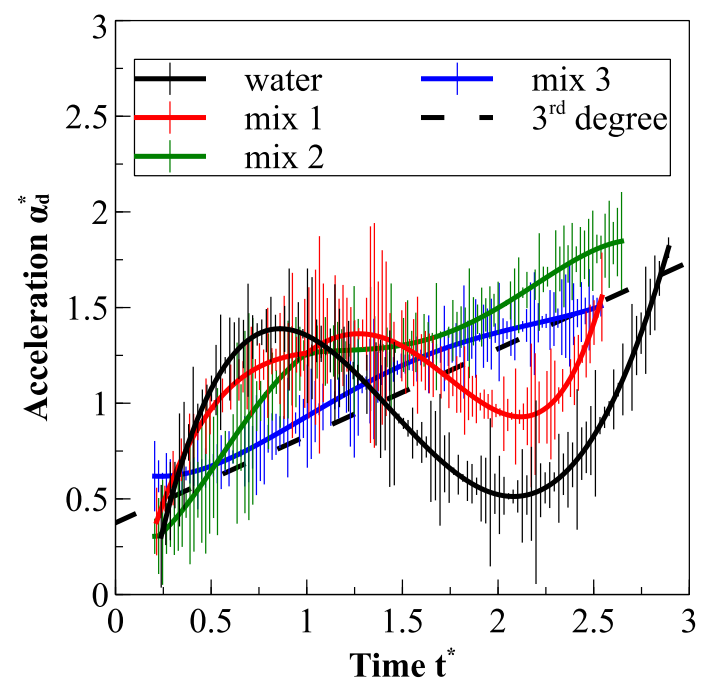

(c) Acceleration $\alpha_{d}^{*}$

Figure 5. Comparison of the temporal evolution of the (a) displacement $x_{d}^{*}$, (b) velocity $u_{d}^{*}$ and (c) acceleration $\alpha_{d}^{*}$ of oblate droplets $(A R=1.2)$ under the no breakup regime for water, $50 \%, 80 \%$ and $97 \%$ glycerol solutions with the $3^{\text {rd }}$ degree fitting curve [4] 


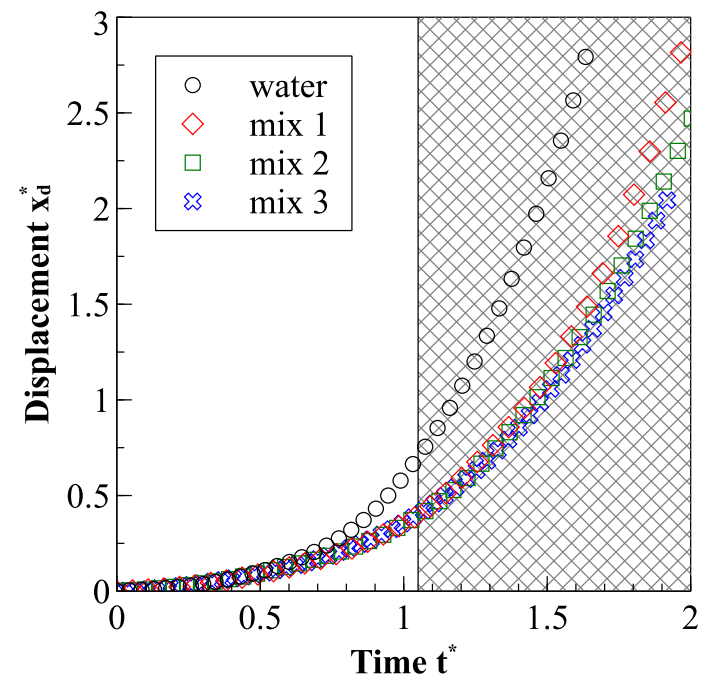

(a) Displacement $x_{d}^{*}$

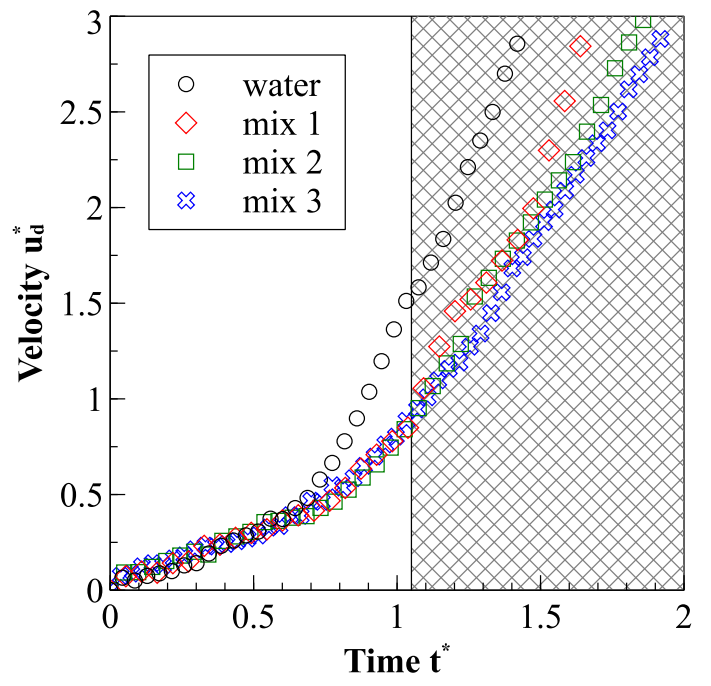

(b) Velocity $u_{d}^{*}$

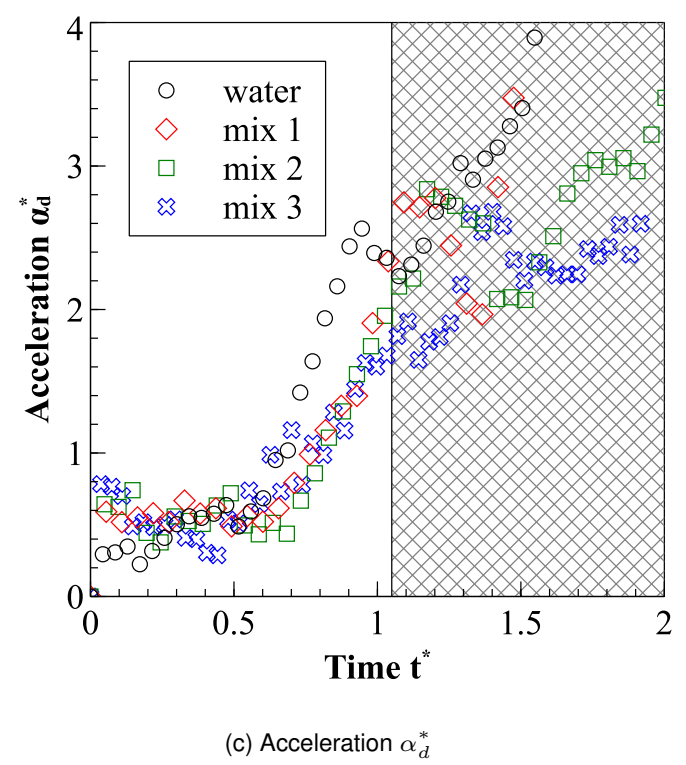

Figure 6. Comparison of the temporal evolution of the (a) displacement $x_{d}^{*}$, (b) velocity $u_{d}^{*}$ and (c) acceleration $\alpha_{d}^{*}$ of the centre of mass of oblate droplets $(A R=1.2)$ under the bag-stamen breakup regime for water, $50 \%, 80 \%$ and $97 \%$ glycerol solutions with the $3^{\text {rd }}$ degree fitting curve [4]

High $W e$ number - Bag-stamen breakup regime

Figure 6 presents the measured displacement $x_{d}^{*}$, and the deduced velocity $u_{d}^{*}$ and acceleration $\alpha_{d}^{*}$ of the droplet centre of mass with time for various liquid viscosities. The gray area of the figures correspond to the breakup initiation time $t_{\text {init }}^{*}$ of the most viscous case and is equal to $t_{\text {init }}^{*}=1.02$; the corresponding breakup initiation time for the least viscous droplet is $t_{\text {init }}^{*}=0.97$.

At short times the effect of increasing viscosity on both displacement and velocity is minor, despite the order of magnitude in the viscosity differences. Detailed differences can be seen in the acceleration values (Figure 6c). The acceleration of the water droplet at time $t^{*}=0$ is smaller than the acceleration for the more viscous droplet due to the higher initial deformation. In later times, due to its higher frontal area the acceleration increases and consequently its velocity increases and its displacement overtake the other droplets. It is of interest to notice that the three more viscous cases have almost the same velocity and displacement and trajectory differentiation starts much latter in time, in fact approximately around the breakup initiation time.

\section{Conclusions}

The role of the initial sphericity of the droplet on the breakup process was experimentally investigated, over a range of $W$ e from 10 to 200 and of the $O h$ number from 0.01 to approximately 4 . It was observed that the breakup modes of initially spherical droplets were in good agreement with the literature but equivalent $W e_{\mathrm{eq}}$ and $O h_{\mathrm{eq}}$ numbers were required to extend the existing morphological classification charts for initially non-spherical as well as spherical 
droplets. For the higher $O h$ numbers $(O h>1)$ not a discernible distinction between breakup modes was possible. The kinematic characteristics of droplets of $A R=1.2$ showed that the liquid viscosity has an important role through the dampening of the internal flow field and two viscosity droplets are accelerated at higher rates due to faster deformation which results in greater frontal area.

\title{
Acknowledgments
}

The authors would like to acknowledge the financial support of Volvo Car Corporation (VCC), Gothenburg, Sweden and EPSRC grant EP/K019732/1.

\author{
Nomenclature \\ $\mathrm{t}$ time [s] \\ $\mathrm{t}^{*}$ dimensionless time [-] \\ AR aspect ratio [-] \\ We Weber number [-] \\ $\mathrm{We}_{e q}$ equivalent Weber number [-] \\ Oh Ohnesorge number [-] \\ $\mathrm{Oh}_{e q}$ equivalent Ohnesorge number [-] \\ $\rho_{g, l} \quad$ gas, liquid density $\left[\mathrm{kg} / \mathrm{m}^{3}\right]$ \\ $u_{g} \quad$ gas velocity $[\mathrm{m} / \mathrm{s}]$ \\ $d_{0} \quad$ volume based droplet diameter [m] \\ $d_{e q} \quad$ equivalent droplet diameter $[\mathrm{m}]$ \\ $\mu_{g, l} \quad$ gas liquid dynamic viscosity [Pa s] \\ $\sigma \quad$ surface tension $[\mathrm{N} / \mathrm{m}]$ \\ $\epsilon \quad$ density ratio [-] \\ $C_{d} \quad$ drag coefficient [-] \\ $x_{d}^{*} \quad$ dimensionless droplet displacement [- \\ $u_{d}^{*} \quad$ dimensionless droplet velocity [-] \\ $\alpha_{d}^{*} \quad$ dimensionless droplet acceleration [-]
}

\section{References}

[1] K. Bergeles, G. Charalampous, Y. Hardalupas, and A. M. K. P. Taylor, "Breakup of Non-spherical Droplets," in 27th Annual Conference on Liquid Atomization and Spray Systems, vol. 1, pp. 4-7, 2016.

[2] E. Villermaux, "Fragmentation," Annual Review of Fluid Mechanics, vol. 39, pp. 419-446, jan 2007.

[3] J. O. Hinze, "Fundamentals of the hydrodynamic mechanism of splitting in dispersion processes," AlChE Journal, vol. 1, no. 3, pp. 289-295, 1955.

[4] M. Pilch and C. Erdman, "Use of breakup time data and velocity history data to predict the maximum size of stable fragments for acceleration-induced breakup of a liquid drop," International Journal of Multiphase Flow, vol. 13, no. 6, pp. 741-757, 1987.

[5] R. Brodkey, Formation of drops and bubbles. Addison-Wesley, 1967

[6] B. Gelfand, "Droplet breakup phenomena in flows with velocity lag," Progress in Energy and Combustion Science, vol. 22, no. 96, pp. 201-265, 1996.

[7] C. Schneider, W. Rasband, and K. Eliceiri, "NIH Image to ImageJ: 25 years of image analysis," Nature Methods, vol. 9, no. 7, pp. 671-675, 2012. 